\title{
ALCOOLISMO: UM ESTUDO ENTRE OS UNIVERSITÁRIOS DAS INSTITUIÇÕES PRIVADAS DE ENSINO DE GUARULHOS
}

\section{ALCOHOLISM: A STUDY AMONG UNIVERSITY STUDENTS OF PRIVATE INSTITUTIONS OF HIGHER EDUCATION OF GUARULHOS}

Recebimento: 26/4/2017- Aceite: 15/05/2017- Publicação: 30/06/2017

Processo de Avaliação: Double Blind Review

\author{
Tiago Crispim da Silva ${ }^{1}$ \\ Bacharel em Administração \\ Centro Universitário ENIAC \\ 218362013@eniac.edu.br
}

\section{Leonam Brito Ribeiro dos Santos \\ Bacharel em Administração \\ Centro Universitário ENIAC \\ leo.jv2012@gmail.com}

\section{Maria Helena Veloso Salgado \\ Professora do Centro Universitário ENIAC \\ maria.helena@eniac.edu.br}

\section{RESUMO}

O consumo em excesso de bebidas alcoólicas entre os universitários da região de Guarulhos está em constante crescimento, pois se observa que os mesmos estão deixando de lado a procura da qualificação profissional para buscarem a diversão em bares e baladas locais próximos às instituições de ensino. $\mathrm{O}$ artigo aborda em fundamentação teórica o que é alcoolismo, causas e efeitos colaterais físicos e psicológicos e as consequências, comprometendo á saúde do estudante. Por meio da aplicação do questionário físico e virtual na instituição de ensino tornou-se primordial para aprofundar no estudo epidemiológico, por conta dos auxílios de livros acadêmicos relacionados à Organização Mundial da Saúde e também perguntas fechadas quantitativas e qualitativas elaboradas pelos autores do artigo. Com a análise de resultados, é possível identificar o número de universitários que podem ser considerados dependentes químicos, entretanto, existem estudantes que ingerem e não são considerados alcoólatras, também dados que apresentam os motivos do consumo, os tipos de bebidas ingeridas pelos alunos, tais como destilados, bebidas ice, cerveja e vinhos, e quantidade de doses ingeridas ao dia, onde dentro desse cenário, a preocupação é grande e os mesmos têm a

\footnotetext{
${ }^{1}$ Autor para correspondência: Centro Universitário ENIAC: R. Força Pública, 89 - Centro, Guarulhos - SP, Brasil. CEP: 07012-030.
} 
consciência dos males que podem trazer e os tornarem dependentes do álcool.

Palavras chave: Alcoolismo. Bebidas alcoólicas. Dependentes químicos.

\begin{abstract}
The excessive consumption of alcoholic beverages among university students in the region of Guarulhos is constantly growing, as it is observed that they are leaving aside the demand for professional qualification to seek fun in local bars and parties near schools. The article deals with theoretical reasons for alcoholism, causes and physical and psychological side effects and consequences, compromising the health of the student. Through the application of the physical and virtual questionnaire in the educational institution, it became essential to deepen in the epidemiological study, due to the aid of academic books related to the World Health Organization and also closed quantitative and qualitative questions elaborated by the authors of the article. With the analysis of results, it is possible to identify the number of university students that can be considered as chemical dependents; however, there are students who drink and are not considered alcoholics, also data that shows the reasons for consumption, the types of drinks ingested by the students, such as distillates, ice drinks, beer and wine, and the amount of doses taken each day, where within this scenario, the concern is great and they are aware of the evils that can bring and make them dependent on alcohol.
\end{abstract}

Key words: Alcoholism. Alcoholic drinks. Chemical dependents.

\title{
INTRODUÇÃO
}

Este artigo pretende analisar o consumo do álcool entre os alunos universitários, assunto relevante na sociedade, todavia, muitos estão deixando de estudar para frequentar bares e baladas em locais próximos às instituições, pesquisaremos a principal causa do consumo excessivo entre os estudantes.

Qual o número de alunos universitários do município de Guarulhos que podem ser caracterizado como alcoólatras?

Que fatores provocam os estudantes universitários a ingerir bebidas alcoólicas em determinada quantidade de acordo com a Organização Mundial de Saúde OMS?

As hipóteses do primeiro problema podem ser detectadas entre a maior parte do público universitário que realmente estão passando a depender do álcool, caracterizados entre os estudantes que ingerem bebidas alcoólicas e que são considerados dependentes químicos e outro grupo que consomem socialmente, porém não considerados alcoólatras de acordo com a dosagem ingerida.

As hipóteses do segundo problema são que os próprios estudantes têm a 
consciência dos males que o álcool causa no ambiente familiar, na vida financeira e até mesmo profissional.

A proposta desse estudo tem como objetivo identificar o número e os motivos que levam os universitários das instituições de ensino superior de Guarulhos a consumir bebidas alcoólicas.

Ademais também de:

- Caracterizar o alcoólatra e os principais efeitos colaterais e causas a saúde física e mental;

- Quantificar o número de universitários alcoólatras por amostragem no município de Guarulhos segundo a OMS e;

- Listar as principais causas que levam os universitários ao consumo de bebida alcoólica.

Como o alcoolismo é considerado uma grave doença que afeta a população brasileira, é significativa a abordagem entre os jovens das instituições de ensinos superiores sobre os principais efeitos que o abuso excessivo pode causar no comportamento físico e psíquico, pois segundo a Organização Mundial da Saúde (2004) citado por Andrade, Anthony e Silveira (2009), afirma que o alcoolista é considerado uma pessoa que consome compulsivamente, onde esta dependência é acompanhada de problemas mentais, físicos, relações com outros indivíduos e comportamento social e econômico.

De fato, Laranjeira e Pinsky (2012) relata que o álcool está presente em todos os ambientes ocasionais, considerados agradáveis ao público (Comemorações, alegrias, relaxamento).

Para Andrade, Anthony e Silveira (2009) o ser humano nunca está satisfeito com a quantidade ingerida, visto que é um dos fenômenos da dependência e o desejo obsessivo pelo álcool, ou seja, o consumo em excesso causa nas pessoas sintomas de alucinação, o corpo cansado e para aumentar a disposição, começam a beber novamente.

\section{REFERENCIAL TEÓRICO}

Jovens e estudantes veem enfrentando uma batalha contra o alcoolismo, pois os universitários estão consumindo cada vez mais entre diversos tipos de 
bebidas alcoólicas, tais como destilados, cervejas, bebidas ice e vinho, deixandoos reféns deste mal que tiram a tranquilidade de si próprio e também das pessoas que estão em volta. O número de universitários que deixam de absorver conhecimentos em agregar na sua vida pessoal e profissional para buscar diversão através de bebidas alcoólicas está em constante crescimento, certamente Andrade, Anthony e Silveira (2009) aborda que jovens acima de 15 anos já iniciam o consumo de bebidas alcoólicas, uma estimativa crescimento anual de 2 bilhões da população mundial que são dependentes químicos.

Quando um indivíduo começa a ingerir em consumos abusivos, automaticamente os efeitos colaterais do álcool começam a se apresentar, e conforme Karren et. al (2013) os efeitos são:O álcool é um depressor, afeta a capacidade de julgamento, visão, tempo de reação e coordenação. Quando ingerido com outros depressores, o resultado pode ser amplificado para além dos efeitos de uma droga ou substância isolada e em quantidades muito elevadas, o álcool pode paralisar o centro respiratório do cérebro e causar morte.

Após o quadro de dependência química, inicia o processo de sintomas durante e posteriormente, sendo caracterizados pelos sintomas psicológicos e físicos.

A síndrome de dependência do álcool SDA abordado por Mari e Kielling (2013) refere-se a uma síndrome clínica causada pelos sinais comportamentais, fisiológicos e cognitivos e a síndrome de abstinência são caracterizados por sinais e também sintomas psíquicos e físicos que apresentam resultados da diminuição ou pausa do uso do álcool.

Com toda certeza Andrade, Anthony e Silveira (2009, p. 71) afirmam que os sintomas psicológicos "Caracterizam-se três elementos principais: a alteração do comportamento face ao álcool, a perda de controle e o desejo intenso de consumilo.", enfatizando, esses sintomas são acompanhados pela abstinência, gerando aquela ansiedade para consumir cada vez mais e obedecendo ao seu consciente. Além disto, Pedrosa (2013) enfatiza que o consumo pode aumentar a possibilidade do alcoólatra a obter depressão, isso ocorre pelas propriedades químicas causadas pelo álcool.

Os sintomas físicos, que também são chamados de intoxicação, são os resultados do consumo da bebida, ou seja, sinais causados pelo corpo, problemas 
internos quanto externos, sinais que podem ser passageiros ou agravantes. Além disso, Messas (2014) caracterizam os sintomas físicos por inicio de abstinência, como: sudorese, tremores, ansiedades, alteração de humor, irritabilidade, problemas digestivos e psíquicos, assim, dificulta em resolver problemas do cotidiano e deixando o usuário fragilizado, impossibilitando no relacionamento interpessoal.

Durante o período constante de experimentação e seus sintomas, surge às consequências do alcoolismo, como doenças e traumas, sendo que Laranjeira e Pinsky (2012) destacam as doenças hepáticas como fígado gorduroso, hepatite alcoólica e a cirrose; doenças e cardiovasculares como a cardiomiopia alcoólica, arritmias, infarto, trombose, hipertensão, problemas na gestação e doenças como o câncer.

Diehl et. al (2011) aborda que os tratamentos variam de acordo do estado do paciente, onde o regime de tratamento pode ocorrer em comunidades terapêuticas (Clinicas ou ONG's), medicamentos, ambulatorial/hospitalar-dia e hospitalar em sistema de internação.

A quantidade de dependentes químicos esta aumentando drasticamente, através de pesquisas, o nível de consumidores de bebida alcoólica esta acima da média, segundo Pulcherio, Bicca e Silva (2011) o trauma e risco de uma pessoa falecer podem ocorrer devido à cirrose, acidentes como carro, suicídios, homicídios, doenças malignas e hemorrágicas cerebrovasculares.

\section{METODOLOGIA}

As pesquisas se desenvolvem a partir de uma revisão bibliográfica dos assuntos tratados dentro do tema, tais como alcoolismo entre os estudantes e a influência do álcool na vida cotidiana. Nesta ótica,Gil (2008) refere-se ao desenvolvimento de pesquisa com materiais já elaborados, desde livros e artigos de forma qualitativa, contendo sua principal vantagem do leitor obter uma série de conhecimentos amplos para o desenvolvimento da pesquisa.

A parte prática desse estudo é uma pesquisa quantitativa realizada através da aplicação de questionário em universitários da região de Guarulhos. Para identificar o motivo que levam os mesmos ao consumo de bebidas alcoólicas, 
Cervo, Barros e Silva (2007) declaram que o questionário é um instrumento bastante utilizado para coletar dados, possibilitando a medição com mais clareza o que se deseja, ou seja, refere-se ao conjunto de questões fechadas ou abertas relacionadas com um problema central.

\section{COLETA DE DADOS}

A ideia inicial foi à aplicação de questionário na Faculdade Eniac e mais duas instituições de ensino superior, tratando-se de nomes fictícios Faculdade Renovar e Faculdade de empreendedorismo de Guarulhos.

A parte prática da pesquisa se desenvolve com a aplicação de questionário, sendo adaptada com o objetivo em avaliar o comportamento do universitário em relação ao alcoolismo, com respostas fechadas e em três etapas:

A primeira etapa na caracterização de dados variáveis qualitativos, como: Faixa etária, sexo, estado civil, quantidade de filhos, instituição de ensino e o tipo de curso que o estudante esta matriculada (Ciências humanas e ciências exatas). O campo "nome do aluno" não foi incluso, pelo fato do questionário ser anônimo, mantendo o sigilo do universitário no ato do preenchimento e tabulação dos dados.

A segunda e terceira etapa consiste em oito perguntas, parte desse questionário validado em pesquisa por Laranjeira et al. (2007) e outras criadas pelos autores do artigo, que estão relacionadas ao alcoolismo, a frequência e quantidade do consumo de bebidas alcoólicas, de acordo com a OMS.

A coleta de dados foi realizada com 371 questionários em salas de aula, com a prévia autorização de professores de cada turma e o contato inicial com estudantes através de questionários físicos e também eletrônico (Google forms).

Como não houve um termo de consentimento livre e esclarecido, a abordagem focou nas informações que o objetivo do questionário era de extremo sigilo, para não comprometer a resposta que aluno cedeu sobre o tema e os dados para o desenvolvimento do artigo.

Nas demais instituições foram apresentadas carta timbrada de autorização para aplicação do questionário aos alunos, entretanto, as mesmas indeferiram a coleta de dados até a entrega do artigo.

Todas as respostas do questionário físico foram repassadas para ferramenta 
do googleforms, facilitando a análise de resultados em gráficos, exceto 32questionários que não foram preenchidos nos campos solicitados, assim, sendo invalidados para o estudo.

\section{ANÁLISE DE RESULTADOS}

O estudo epidemiológico contou com a participação de 339 universitários da instituição de ensino Eniac, tendo a prevalência do público masculino 204 universitários (60.2\%) e feminino 135 universitárias (39.8\%) com idades de 17 anos, 22 estudantes (6.5\%) de 18 a 25 anos, 186 estudantes (59.9\%), de 26 a 35 anos, 82 estudantes(24.2\%), de 36 a 45 anos, 36 estudantes (10.6\%) de 46 a 59 anos, 11 estudantes (3.2\%) e acima de 60 anos, 2 estudantes ( $0.6 \%$ ).

O estado civil da grande parte dos estudantes foi relatado entre218 estudantes (64.3\%) solteiros, 105 (31\%) casados. Em relação aos filhos, 236 estudantes (69.5\%) alegaram que não possuem filhos e 48 (14.2\%) tem apenas um filho.

Sobre a área de conhecimento, os dados apresentaram: Ciências humanas 165 estudantes (48.7\%) e ciências exatas 174 (51.3\%), com a maioria das porcentagens destacadas ao $3^{\circ}$ semestre $71(20.9 \%), 9^{\circ}$ semestre $57(16.8 \%), 1^{\circ}$ semestre $54(15.9 \%)$ e $7^{\circ}$ semestre 29 estudantes (8.6\%).

Houve contradições ao analisar os resultados relacionados ao alcoolismo, onde desde a primeira até a última questão, os universitários que afirmaram o "não consumo" responderam de forma irregular, ou seja, dados de entrevistados apresentaram diferenças na tabulação dos dados, entretanto, a quantidade de estudantes que consomem bebidas alcoólicas foi uma surpresa, na grande parte caracterizada em consumo ocasional $(28 \%)$, raramente $(13.9 \%)$ e muito frequente (7.1\%), tendo o reconhecimento e a consciência dos males que a bebida pode causar durante e após o consumo (57.8\%), conforme demonstrado na tabela 1 : 
Tabela 1 -Consome bebida alcoólica.

\begin{tabular}{|c|c|c|}
\hline & Quantidade de Universitários & Porcentagem \\
\hline Muito Frequente (Bebe todos os dias) & 24 & $7.1 \%$ \\
\hline Frequentemente (1-4 vezes por semana) & 27 & $8 \%$ \\
\hline Ocasionalmente (1-3 vezes mês/local) & 95 & $28 \%$ \\
\hline Raramente (Bebe menos de 1 vez ao mês) & 47 & $13.9 \%$ \\
\hline Abstinente (Bebe menos de uma vez ao ano) & 11 & $3.2 \%$ \\
\hline $\begin{array}{l}\text { Não consumo mais bebidas alcoólicas } \\
\text { Não bebo }\end{array}$ & $\begin{array}{c}18 \\
117\end{array}$ & $\begin{array}{c}5.3 \% \\
34.5 \%\end{array}$ \\
\hline TOTAL & 339 & $100 \%$ \\
\hline
\end{tabular}

Fonte: Dados da pesquisa ( 2016)

Aqueles que responderam que não consomem mais bebidas alcoólicas, que gerou no total de 18 estudantes $(5.3 \%),(47.4 \%)$ relataram que pararam por vontade própria.

Ao verificar sobre qual o motivo eles consomem bebidas alcoólicas, 143 (42.2\%) estudantes relataram que bebem por diversão, 25 (7.4\%) por conta da influência de amigos, 23 (6.8\%) por influência de familiares e10 (2.9\%) pelo vício.

Dentre os gastos mensais com bebidas, dados apontaram que 110 alunos (32.4\%) gastam até $R \$ 50,00$ e 30 (8.8\%) de $R \$ 50,01$ a 100,00 e também (8.8\%) acima de $R \$ 200,00$, o que leva a curiosidade em saber qual tipo de bebida que eles consomem.

A Alcoolemia para Rasslan (2016) é uma concentração de álcool no sangue, causado pelos efeitos colaterais de acordo com a miligrama por decilitro $(\mathrm{mg} / \mathrm{dl}) \mathrm{de}$ álcool ingerida e além disso, Andrade, Anthony e Silveira (2009) informam que com as unidades padrões, cada unidade alcoólica possui cerca de 8 a $14 \mathrm{~g}$ de etanol puro, considerando que muitos destes estudantes não tem conhecimento desta informação.

Segundo apresentado na tabela 2, a cerveja foi um índice alto entre essa avaliação de consumo, sendo considerados 130 estudantes (38.3\%), contra 93 (27.4\%) de bebidas destiladas, como cachaça, whisky, vodca, conhaque e rum, 88 (26\%) ao consumo de vinho e $77(22.7 \%)$ ao consumo de vinho na pergunta de múltipla escolha. 
Tabela 2 - O tipo de bebida que o universitário consome (Múltipla escolha)

\begin{tabular}{lcc}
\hline & $\begin{array}{c}\text { Quantidade de } \\
\text { Universitários }\end{array}$ & Porcentagem \\
Cerveja & 130 & $38.3 \%$ \\
Vinho & 88 & $26 \%$ \\
Bebidas "Ice" & 77 & $22.7 \%$ \\
Destilados (Cachaça, Whisky, vodca, conhaque, & 93 & $27.4 \%$ \\
rum) & 139 & $41 \%$ \\
Não bebo & & \\
\hline TOTAL & & \\
\hline
\end{tabular}

Fonte: Dados da pesquisa ( 2016)

Com relação à idade inicial do consumo, 109 (32.2\%) dos estudantes afirmaram que começaram a beber entre 15 a 17 anos e 64 (18.9\%) entre 18 a 28 anos.

Uma porcentagem menor, porém prevaleceu uma preocupação na análise, 28 estudantes, cerca de $8.3 \%$ começaram a ingerir dos seus 12 á 14 anos, fato que se repetem no nosso cotidiano, e que a lei $n^{\circ} 8.069$, de 13 de julho de 1990.do estatuto da criança e do adolescente e dá outras providências, o artigo 244.

\footnotetext{
Vender, fornecer, servir, ministrar ou entregar, ainda que gratuitamente, de qualquer forma, a criança ou a adolescente, bebida alcoólica ou, sem justa causa, outros produtos cujos componentes possam causar dependência física ou psíquica: (BRASIL, 1990, p. 1).
}

Para identificar o alcoólico em excesso, Laranjeira et. al (2007) considera que o beber binge é o alto volume de consumo em um curto prazo de tempo, este ato é considerado muito perigoso, podendo causar danos físicos, mentais e sociais, tendo base em quantidade de 5 doses para homens e 4 doses para mulheres em um único dia.

Os dados abaixo apontam na tabela 3 que 46 universitários (13.6\%) consomem de 5 a 11 doses e 19 (5.6\%) universitários consomem acima de 12 doses diárias. Este excesso de consumo de bebidas por dia gera uma preocupação não só para os seus familiares e pessoas próximas, mas também a si mesmo, podendo causar danos a sua saúde ou levando até a morte. 
Tabela 3 - Quantidade de doses consumidas diariamente.

“Uma dose corresponde, na média, a uma latinha de cerveja ou chope de $350 \mathrm{ml}$, uma taça de vinho de $90 \mathrm{ml}$, uma dose de destilados de $30 \mathrm{ml}$, uma lata ou uma garrafa pequena de qualquer bebida "ice"."

Cada dose contém cerca de $10-12 \mathrm{~g}$ de alcool.

Até duas doses

De 3 a 4 doses

De 5 a 11 doses

Acima de 12 doses

Não bebo
Quantidade de Universitários

83

45

46

19

146
Porcentagem

$24.5 \%$

$13.3 \%$

$13.6 \%$

$5.6 \%$ $43.1 \%$

Fonte: Dados da pesquisa ( 2016)

Ressalta-se que é importante analisar e conferir todas as informações, pois os dados informados convergem para o alcance dos objetivos e validação das hipóteses apresentadas.

\section{CONSIDERAÇÕES FINAIS}

Apesar da delimitação em aplicar os questionários em outras faculdades e também as poucas contradições encontradas na análise de dados obtida entre estudantes da instituição de ensino Eniac, a quantidade de alunos que consomem bebidas alcoólicas é surpreendente, mesmo com horários flexíveis de aulas, sem intervalos e bares aos arredores da instituição, o estudo epidemiológico aponta que 204 alunos, ou seja, 60.2\% tem influência do álcool em suas vidas independente se o consumo é muito frequente ou até mesmo abstinente, e 196 destes afirmaram que ao ingerir, sabem que o excesso de álcool em seu organismo podem causar sérios danos asaúde, o que foi relatado na segunda hipótese levantada, pois todas as respostas foram validadas e fundamentais para o aprofundamento do estudo.

Ressalta-se que o primeiro problema e hipótese levantada, 65 universitários (19.2\%) dos grupos que ingerem bebidas são considerados alcoólatras de acordo com a Organização Mundial a Saúde por conta do consumo diário acima de 5 doses, sendo que: 5 a 11 doses: 46 alunos (13.6\%) e acima de 12 doses: 19 alunos (5.6\%). A coleta de dados viabiliza também no segundo problema e na hipótese, onde a principal bebida consumida entre os estudantes é a cerveja e que a diversão e influência de amigos levam a cometer este ato.Ao analisar qual o tipo de gênero que consome mais bebidas alcoólicas como binge, observa-se que 51 universitários 
do sexo masculino consomem acima de cinco doses diárias, e dentro deste número, 44 são do curso de ciências exatas, 27 são solteiros e a grande parte ingere por conta da influência de amigos.

Após os dados levantados através do estudo, é de fundamental importância no trabalho de conscientização em salas de aula para os futuros profissionais, no entanto se esses universitários permanecerem neste caminho, certamente a instituição perderá um grande talento por conta do excesso de bebidas alcoólicas.

\section{REFERÊNCIAS}

ANDRADE, Arthur Guerra de (ed.); ANTHONY, James C. (ed.); SILVEIRA, Camila Magalhães (co-ed.). Álcool e suas consequências: uma abordagem multiconceitual. Barueri, SP: Minha Editora, 2009.

BRASIL. Lei № 8.069, de 13 de julho de 1990.do estatuto da criança e do adolescente e dá outras providências, o artigo 244.: Disponível em <http://www.planalto.gov.br/ccivil 03/leis/L8069.htm> Acesso em 15 maio 2016.

CERVO, Amado Luiz; BERVIAN, Pedro Alcino; SILVA, Roberto da. Metodologia científica. 6 ed. São Paulo: Pearson Prentice Hall, 2007

DIEHL, Alessandra et. al. Dependência Química: Prevenção, tratamento e políticas públicas - Dados eletrônicos. Porto Alegre: Artmed Editora, 2011.

GIL, Antônio Carlos. Métodos e técnicas de pesquisa social. 6 ed. São Paulo: Atlas, 2008.

KARREN, Keith j. [et al]; Primeiros socorros para estudantes [tradução Patricia Fonseca Pereira e Douglas Arthur Omena Futuro]. 10 ed. Barueri, SP: Manole, 2013

LARANJEIRA, Ronaldo. et al. I Levantamento Nacional sobre os padrões de consumo de álcool na população brasileira. Brasília: Secretaria Nacional antidrogas, 2007.

LARANJEIRA, Ronaldo; PINSKY, Ilana; O Alcoolismo.9 ed. São Paulo: Contexto, 2012.

MARI, Jair de Jesus; KIELLING, Christian (ed.) Psiquiatria na prática clínica. Barueri, SP: Manole, 2013.

MESSAS, Guilherme. Álcool e drogas: uma visão fenômeno-estrutural. São Paulo: Casa do Psicólogo, 2014.

PEDROZA, José. Álcool, Uso e Abuso. Natal: Clube dos autores, 2013. 
PULCHERIO, Gilda; BICCA, Carla e SILVA, Fernando Amarante (org.) Álcool, outras drogas e informação: $O$ que cada profissional precisa saber. 2. ed. São Paulo: Casa do Psicólogo, 2011.

RASSLAN, Zied (Coord). Medicina de urgência, Barueri, SP: Manole, 2016. 